\title{
Parasite histones mediate blood-brain barrier disruption in cerebral malaria
}

\author{
Authors: Christopher Moxon, ${ }^{\mathrm{A}}$ Yasir Alhamdi, ${ }^{\mathrm{B}}$ Janet Storm, ${ }^{\mathrm{C}}$ Julien Toh, ${ }^{\mathrm{D}}$ Joo Yeon Ko, ${ }^{\mathrm{E}}$ George Murphy, ${ }_{\mathrm{C}}$ \\ Terrie Taylor, ${ }^{\mathrm{G}}$ Karl Seydel, ${ }^{\mathrm{G}}$ Guozheng Wang, ${ }^{\mathrm{B}}$ Guillermo García-Car, ${ }^{\mathrm{F}}$ Malcolm Molyneux, ${ }^{\mathrm{C}}$ Alister Craig, ${ }^{\mathrm{C}}$ \\ Simon Abrams ${ }^{B}$ and Cheng-Hock Toh ${ }^{B}$
}

\section{Introduction}

Cerebral malaria (CM) is a severe complication of Plasmodium falciparum infection. Despite effective antimalarial drugs, $10-20 \%$ of children developing CM die, contributing to 400,000 malarial deaths per year, mostly in children in sub-Saharan Africa. ${ }^{1,2}$ A defining feature of CM is the cytoadherence of $P$ falciparum-infected erythrocytes (IE) to vascular endothelial cells and their sequestration in the microvasculature, which is observed by retinopathy. Microvascular thrombosis and blood-brain barrier breakdown are key components of CM pathogenesis. ${ }^{3,4}$ We have previously demonstrated that elevated circulating histones, released from damaged host cells, bind to cell membranes and cause coagulation activation, platelet aggregation, microvascular thrombosis and vascular leak in critically ill patients. ${ }^{5-8}$ The aim of this study was to investigate whether histones are released from parasites to play a pathological role in CM.

\section{Materials and methods}

Children aged 6 months - 16 years were recruited at Queen Elizabeth Central Hospital, Blantyre, Malawi between January 2010 and August 2011; inclusion criteria were described previously. ${ }^{9}$ Children who met World Health Organization criteria for CM underwent funduscopic examination by an ophthalmologist: characteristic retinal changes are strongly associated with sequestration of IE in the brain and distinguish children with stringently defined retinopathypositive (ret-pos) CM from those with retinopathy-negative (ret-neg) CM, to which malaria makes a variable contribution to coma aetiology. ${ }^{1}$ Healthy controls were children attending elective surgery. Circulating histones were quantified by Western blot, and mass spectrometry was used to determine

Authors: ${ }^{A}$ University of Glasgow, Glasgow, UK; ${ }^{B}$ University of Liverpool, Liverpool, UK; ' Liverpool School of Tropical Medicine,

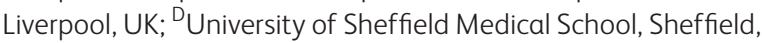
UK; ${ }^{E}$ Hanyang University College of Medicine, Seoul, South Korea; ${ }^{\mathrm{F}}$ Harvard Medical School, Boston, USA; ${ }^{G}$ Michigan State University, East Lansing, USA the origin of histones. Endothelial toxicity and leakage assays were performed using primary human brain microvascular endothelial cells. Magnetic resonance imaging (MRI) images acquired on admission were scored independently by two radiologists, blinded to patient details. ${ }^{3}$ Formalin-fixed post-mortem brain tissues from Malawian children with fatal encephalopathic illness were collected and stained for histones and fibrinogen.

\section{Results and discussion}

Using Western blot and mass spectrometry, we demonstrate that extracellular histones are significantly elevated in the circulation of CM patients compared with controls, with over $50 \%$ released from parasites. Circulating histones were significantly elevated in ret-pos CM compared with retneg CM. Serum from patients with ret-pos CM or purified $P$ falciparum histones are toxic to cultured human brain endothelial cells and cause disruption of barrier function. This can be reversed by anti-histone antibodies and nonanticoagulant heparin. On post-mortem brain sections of patients with CM, we found that histones are co-localised with $P$ falciparum parasites sequestrated within the brain vasculature, suggesting that histones may be locally released from parasitic breakdown. Histone staining on the luminal vascular surface is strongly associated with thrombosis and leakage, indicating coagulation activation and endothelial disruption. High levels of circulating histones were significantly associated with the extent of brain swelling on MRI, providing further clinical evidence for the role of histones in this process.

\section{Conclusion}

Our data strongly suggest that parasite histones play key roles in thrombosis and swelling in the brain, processes implicated in the causal pathway to death in CM. Neutralising histones with agents such as non-anticoagulant heparin holds great promise to prevent the development of $\mathrm{CM}$ and reduce mortality of children with malaria.

\section{Conflicts of interest}

None declared. 


\section{References}

1 Taylor TE, Fu WJ, Carr RA et al. Differentiating the pathologies of cerebral malaria by postmortem parasite counts. Nat Med 2004;10:143-5.

2 World Health Organization. World malaria report 2017. Geneva: WHO, 2017. www.who.int/malaria/publications/world-malariareport-2017/en [Accessed 10 December 2019].

3 Seydel KB, Kampondeni SD, Valim C et al. Brain swelling and death in children with cerebral malaria. $N$ Engl J Med 2015;372:1126-37.

4 Mohanty S, Benjamin LA, Majhi M et al. Magnetic resonance imaging of cerebral malaria patients reveals distinct pathogenetic processes in different parts of the brain. mSphere 2017;2:e00193-17.
5 Alhamdi Y, Abrams ST, Lane S, Wang G, Toh CH. Histoneassociated thrombocytopenia in patients who are critically ill. JAMA 2016;315:817-9.

6 Abrams ST, Zhang N, Dart C et al. Human CRP defends against the toxicity of circulating histones. J Immunol 2013;191:2495-502.

7 Abrams ST, Zhang N, Manson J et al. Circulating histones are mediators of trauma-associated lung injury. Am J Respir Crit Care Med 2013;187:160-9.

8 Alhamdi Y, Abrams ST, Cheng Z et al. Circulating histones are major mediators of cardiac injury in patients with sepsis. Crit Care Med 2015;43:2094-103.

9 Moxon CA, Wassmer SC, Milner DA Jr et al. Loss of endothelial protein $\mathrm{C}$ receptors links coagulation and inflammation to parasite sequestration in cerebral malaria in African children. Blood 2013;122:842-51. 